\title{
Isolated Pneumopericardium in a Preterm Infant on Nasal Biphasic Positive Airway Pressure
}

\author{
Zeynep Alp Unkara, d, Ozge Gemicib ${ }^{\mathrm{b}}$, Asli Memisoglu ${ }^{\mathrm{a}}$, Figen Akalin ${ }^{\mathrm{c}}$, Hulya Bilgen ${ }^{\mathrm{a}}$, Eren Ozek ${ }^{\mathrm{a}}$
}

\begin{abstract}
Different modalities of non-invasive ventilation (NIV), one of which is nasal biphasic positive airway pressure (nBiPAP), are used both after extubation and as an alternative to intubation in suitable cases. Just as mechanical ventilation (MV), NIV can be complicated by air leak syndromes. Pneumopericardium (PPC), especially if isolated, is the rarest of these conditions. We present a case of isolated PPC in a preterm newborn as a complication of nBiPAP.
\end{abstract}

Keywords: Pneumopericardium; Preterm; Non-invasive ventilation; nBiPAP

\section{Introduction}

Respiratory distress syndrome (RDS) is almost exclusively a disease of the preterm neonate. Although the use of mechanical ventilation (MV) has been life-saving throughout the years of its use, being intubated and connected to a ventilator is by itself a risk factor for ventilator-induced lung injury [1]. Noninvasive ventilation (NIV) modalities, namely nasal continuous airway pressure (nCPAP), nasal intermittent positive pressure ventilation (NIPPV) and nasal biphasic positive airway pressure (nBiPAP) are being increasingly used both to increase the success of extubation, and to avoid the initial trauma of intubation and MV [1].

Biphasic positive airway pressure ventilation differs from NIPPV in that it uses a variable jet flow system. Instead of

\footnotetext{
Manuscript accepted for publication November 25, 2014

aNeonatology, Department of Pediatrics, Marmara University Faculty of Medicine, Istanbul, Turkey

bDepartment of Pediatrics, Marmara University Faculty of Medicine, Istanbul, Turkey

'Pediatric Cardiology, Department of Pediatrics, Marmara University Faculty of Medicine, Istanbul, Turkey

${ }^{\mathrm{d} C o r r e s p o n d i n g ~ A u t h o r: ~ Z e y n e p ~ A l p ~ U n k a r, ~ M a r m a r a ~ U n i v e r s i t e s i ~ P e n d i k ~}$ Egitim ve Arastirma Hastanesi, Fevzi Cakmak Mh. Mimar Sinan Cd. No. 41 Pendik 34899, Istanbul, Turkey. Email: md.zeynepalp@gmail.com
}

doi: http://dx.doi.org/10.14740/ijcp173w forced ventilatory breaths, it allows the patient to breathe freely while providing two different levels of pressure with preset time intervals and rate $[1,2]$. Causing similar complications as those of CPAP (including air leak syndromes, over distension of lungs, nasal irritation, etc.), nBiPAP improves tissue oxygenation, carbon dioxide $\left(\mathrm{CO}_{2}\right)$ clearance and decreases the effort of breathing [2]. Thus, in cases where CPAP is inadequate to provide the necessary ventilatory support, $\mathrm{nBiPAP}$ may be used.

Pulmonary air leak syndromes are complications of MV and NIV [3, 4]. Pneumopericardium (PPC) is rare and usually occurs with other simultaneous air leaks, such as pneumothorax (PT), pulmonary interstitial emphysema, and pneumomediastinum [4]. Even though there are cases of spontaneous PPC, the majority of cases are associated with neonatal resuscitation, RDS, MV or CPAP. The most probable pathology for isolated PPC is thought to be that the air from ruptured alveoli flows along the course of main pulmonary vessels and enters the pericardium through a site of thin mesothelium where parietal pericardium reflects onto visceral pericardium, an area lacking pericardial collagenous tissues [3].

We report a case of isolated PPC in a preterm newborn on nBiPAP. To our knowledge, this is the first case of PPC reported as a complication of nBiPAP.

\section{Case Report}

The female infant weighing $1,820 \mathrm{~g}$ was the second-born twin, born to a 28 -year-old, gravida 2 , para 1 mother following a 31 -week gestation. The pregnancy was uncomplicated until the onset of premature labor. First dose of antenatal betamethasone was given to the mother when she first applied to the emergency room with premature contractions. Three hours thereafter, dichorionic diamniotic twins were born by cesarean section.

The baby did not cry immediately after birth, had gasping and intercostal retractions, and was cyanotic. Since heart rate was above 60 beats/min, only positive pressure ventilation was applied via a T-piece resuscitator for $60 \mathrm{~s}$, after which cyanosis and gasping resolved, but retractions remained. The Apgar score was 5,7 and 8 for the 1,5 and $10 \mathrm{~min}$, respectively. The patient was transferred to the neonatal intensive care unit (NICU) on CPAP. Cord blood gas sampling showed pH: 7.27, 


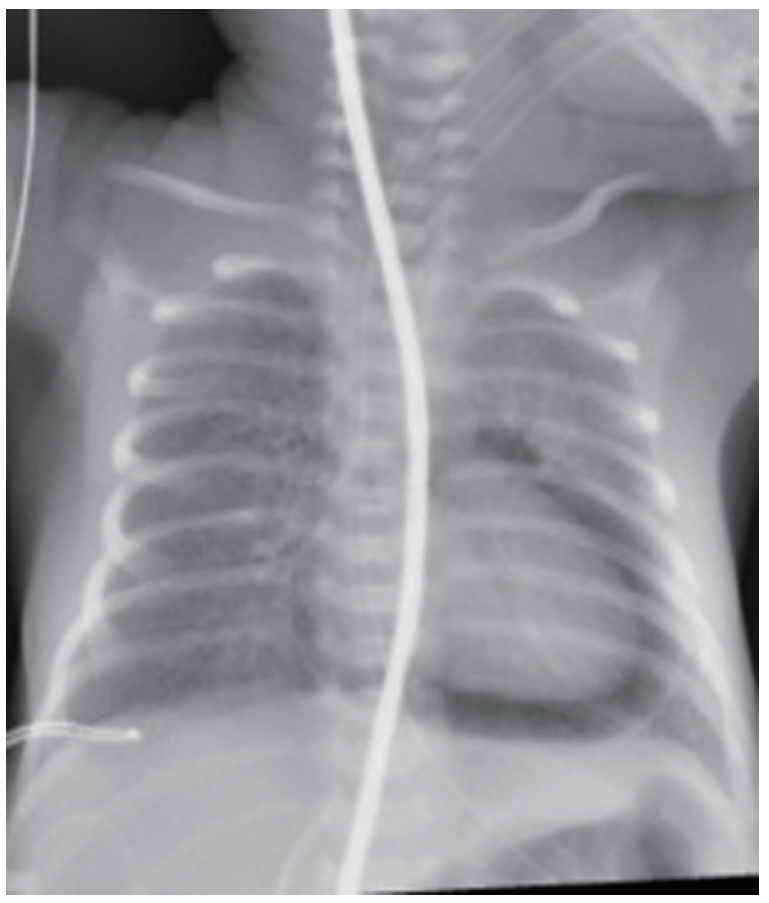

Figure 1. The first chest X-ray revealing PPC without any other air leak.

$\mathrm{pCO}_{2}: 51.4 \mathrm{~mm} \mathrm{Hg}, \mathrm{HCO}_{3}: 22.6 \mathrm{mmol} / \mathrm{L}$, base excess: -4.4 $\mathrm{mmol} / \mathrm{L}$ and lactate: $2.4 \mathrm{mmol} / \mathrm{L}$.

The vital parameters upon admission to NICU showed a heart rate of 156 beats/min, respiratory rate of 68/min with intercostal retractions, nasal flaring and grunting. Capillary refill

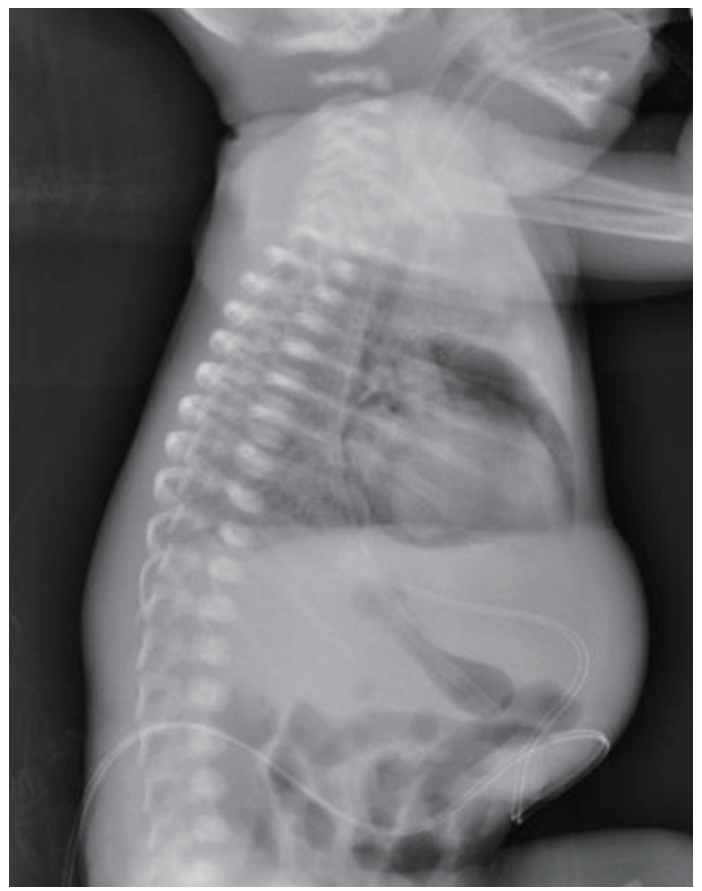

Figure 2. Lateral view of PPC.

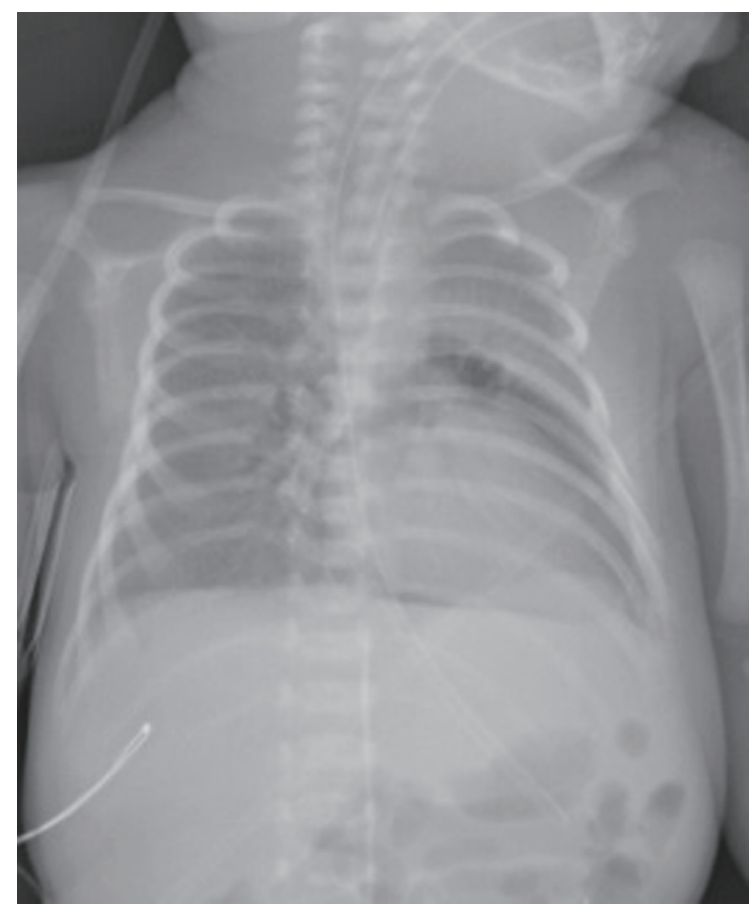

Figure 3. Partially resorbed PPC.

was 2 s, and arterial blood pressure was 59/36 mm Hg (between 50th and 95th percentile of the normal limits based on gestational age). Initial chest X-ray was consistent with mild RDS; however, since retractions and grunting receded gradually and the patient was stable on $6 \mathrm{~cm} \mathrm{H} \mathrm{H}_{2} \mathrm{O}$ CPAP with $\mathrm{FiO}_{2}$ of $25 \%$, she was not given surfactant.

After $18 \mathrm{~h}$ of uneventful course, intercostal retractions reemerged together with tachypnea. Capillary blood gas analysis and chest X-ray were both normal, and the patient was switched to nBiPAP (Infant Flow ${ }^{\circledR}$ SiPAP System, CareFusion) with pressures $10 \mathrm{~cm} \mathrm{H}_{2} \mathrm{O} / 6 \mathrm{~cm} \mathrm{H}_{2} \mathrm{O}$, rate 20 breaths/min and inspiratory time $0.5 \mathrm{~s}$. Twelve hours later while on nBiPAP arterial blood pressure suddenly dropped below 5 th percentile, tacypnea, retractions, tachycardia (182 beats/min) and cutis marmoratus developed. Breath sounds were equally auscultated on both hemithoraces. Capillary blood gas analysis, which was completely normal $2 \mathrm{~h}$ prior to this acute episode, showed respiratory and metabolic acidosis with an increase in lactate level (pH: 7.08, $\mathrm{pCO}_{2}: 60 \mathrm{~mm} \mathrm{Hg}, \mathrm{HCO}_{3}: 13 \mathrm{mmol} / \mathrm{L}$, base excess: $-10 \mathrm{mmol} / \mathrm{L}$, lactate: $5.5 \mathrm{mmol} / \mathrm{L})$. The baby was intubated, saline infusion was started for hypotension and chest $\mathrm{X}$-ray was taken. On chest X-ray, a halo sign around the heart was clearly visible without proof of any other air leaks (Fig. $1,2)$. Echocardiographic examination was non-diagnostic because of the poor visualization due to air interference, which was repeated and found to be normal after resolution of the air leak. Blood pressure and heart rate returned to normal limits after saline infusion, and the baby's condition did not deteriorate any further; thus, pericardiocentesis was not needed. Chest X-ray taken $6 \mathrm{~h}$ later showed partial resolution of PPC (Fig. 3). Thereafter, the infant's clinical condition improved, and respiratory distress resolved quickly. Her subsequent chest 
X-ray showed complete resolution of the PPC.

\section{Discussion}

Isolated PPC is a rare entity and is usually associated with MV, CPAP or neonatal resuscitation [3, 5]. Even though isolated PPC cases have been reported, it usually occurs together with other air leak syndromes, PT being the most common $[4,5]$. In our case, positive pressure ventilation was applied via a Tpiece resuscitator in the delivery room for $60 \mathrm{~s}$, but the initial chest X-ray was negative for any air leak. PPC and thus deterioration of the patient's condition took place $12 \mathrm{~h}$ after she was put on nBiPAP as a complication of this modality.

The treatment method for isolated PPC still remains controversial. Unlike PT, the treatment modality for which is definite, whether to perform pericardiocentesis or not is something the physician has to decide according to the patient's condition. In the absence of cardiac tamponade, isolated and clinically insignificant cases of PPC could be closely monitored without any intervention. Spontaneous resolution is the most common result in such cases [3-5]. Inhalation of $100 \% \mathrm{O}_{2}$, the "nitrogen washout" therapy, has some effect over the resolution of air leaks; however, it is of limited use on infants born earlier than 32 weeks of gestation because of the risk of hyperoxia and associated retinopathy of prematurity [5]. If the patient's condition continues to deteriorate with clinical signs of cardiac tamponade and cardiovascular collapse, invasive treatment methods, such as needle aspiration or tube insertion for continuous drainage, should be performed without further delay $[3,5]$.
Our patient's cardiovascular status recovered after the initial saline infusion, necessitating no further intervention. Therefore, conservative approach with continuous monitoring of oxygen saturation, heart rate and blood pressure was preferred; successive chest X-rays were planned to observe the resorption of pericardial air.

In conclusion, although non-invasive ventilatory support has been demonstrated to be less injurious to preterm lung, it still may cause serious complications. Isolated PPC, which is a rare form of air leak syndromes, can be a complication of nBiPAP.

\section{References}

1. Narasimhan R, Krishnamurthy S. A review of non-invasive ventilation support in neonates. Paediatr Child Health. 2014;24(1):7-11.

2. Migliori C, Motta M, Angeli A, Chirico G. Nasal bilevel vs. continuous positive airway pressure in preterm infants. Pediatr Pulmonol. 2005;40(5):426-430.

3. Mansfield PB, Graham CB, Beckwith JB, Hall DG, Sauvage LR. Pneumopericardium and pneumomediastinum in infants and children. J Pediatr Surg. 1973;8(5):691699.

4. Hook B, Hack M, Morrison S, Borawski-Clark E, Newman NS, Fanaroff A. Pneumopericardium in very low birth weight infants. J Perinatol. 1995;15(1):27-31.

5. Suryawanshi P, Klimek J. Preterm Neonate with Spontaneous Pneumopericardium without any Other Associated Air Leaks. J Clin Diagn Res. 2014;8(1):181-182. 